\title{
Examining the Effects of Teacher and Self-Evaluation of Disruptive Behavior via School-Home Notes for Two Young Children in Kindergarten
}

\author{
Kara E. McGoey, Tana Prodan and Nancy Condit
}

\begin{abstract}
This study replicates and extends previous research in the area of managing the disruptive classroom behavior of young children. The effectiveness of teacher and self-evaluation through schoolhome notes was explored. A combination self-evaluation and school-home note procedure was implemented with two kindergarten boys. A single-subject reversal design was used to analyze direct observations of disruptive behavior. Results indicated a decrease in disruptive behavior associated with the intervention. The intervention procedures were also found to be acceptable by the teacher. The gap between evidence-based interventions and practice was addressed by implementing a combination of efficacious interventions in the participants' natural environment. Implications for practice and further research are presented.

Keywords: Self-evaluation, school-home notes, classroom intervention, early childhood.
\end{abstract}

Children entering kindergarten are expected to be ready to learn. Teachers anticipate that children have readiness skills such as reciting the alphabet, knowing their name, and counting to at least 10. In addition, children entering kindergarten are expected to display age-appropriate social skills and appropriate classroom behavior. This includes paying attention to the teacher, sitting in their seat, and negotiating social situations with other young children. Young children with disruptive classroom behavior fail to meet the goal of entering school ready to learn. Inattention, impulsivity, aggression, noncompliance, and hyperactivity may impede the learning process for the child with disruptive behavior. Additionally, disruptive behavior may create an atmosphere that interferes with the learning of other children, and these behaviors often frustrate the classroom teacher (DuPaul, McGoey, Eckert, \& Van Brakle, 2001). It is estimated that 3-5\% of elementary age children are diagnosed with Attention Deficit/Hyperactivity Disorder, and many children with ADHD exhibit disruptive behavior in the classroom (ADHD; Barkley, 1998).

Behavior management procedures have empirical support for children with ADHD and emotional and behavior disorders (DuPaul \& Eckert, 1997; Pelham, Wheeler, \& Chronis, 1998); however, these interventions have been primarily implemented with older elementary age children or adolescents and are often underutilized in practice (McGoey, Eckert, \& DuPaul, 2000). Literature examining interventions to manage disruptive behavior of young children in the classroom have been limited in scope. Positive reinforcement, response cost, self-monitoring, and school-home note procedures have been implemented with young children, but the literature is lacking empirical support due to small sample sizes and limited replications (McGoey, et al., 2000).

In addition, the interventions that have been shown to be efficacious in the scholarly literature are not always found to be as effective in classrooms (Kratochwill \& Stoiber, 1999; Stoiber \& Kratochwill, 1999). Therefore, applied investigations must explore the efficacy and effectiveness of classroom-based interventions (Kratochwill \& Stoiber; Stoiber \& Kratochwill). This lack of effective interventions for young children with disruptive behavior adds to the void between scientific research and educational 
practice. This study attempts to address the research to practice gap by implementing a multi-component intervention including teacher and self-evaluation via a school-home note in a kindergarten classroom.

Self-management interventions have been implemented to successfully decrease disruptive behavior and increase prosocial behavior of children with emotional and behavior disorders (EBD) (DuPaul \& Stoner, 2003; Mooney, Ryan, Uhingm Reid, \& Epstein, 2005). Self-management techniques often require students to evaluate their own behavior, rate it on a continuum, compare that rating to the rating of the teacher, and access reinforcers for behavior meeting a pre-specified criterion. Mooney and colleagues conducted a comprehensive review of self-management interventions for students with EBD. Their findings indicate effect sizes large in magnitude (range $=1.13-2.71$ ) for all components of selfmanagement packages. In addition, Reid, Trout, and Schartz (2005) conducted a meta-analysis of studies investigating self-regulation interventions for students with ADHD. The analysis included the following interventions under the umbrella terms of self-regulation, self-monitoring, self-monitoring plus reinforcement, self-management, and self-reinforcement. Again, effect sizes were large in magnitude for all four intervention types. However, the majority of studies reviewed in both investigations included participants aged 7 to 12 . Minimal research has been conducted in younger children and adolescents (Mooney et al.).

One example of self-management strategies in young children was investigated by Workman, Helton, and Watson (1982). They investigated the effects of self-monitoring on the sustained schoolwork behavior of a 4 year old referred for difficulties completing school work and noncompliance with teacher requests. The participant was taught to mark a sheet of paper if he was working on an assigned task when a kitchen timer rang. Results indicated an increase in sustained schoolwork behavior and decreased noncompliance. Despite the promising results of this intervention, it is a case study, thus lacking generalizability. Parent involvement and collaboration was also missing in the intervention planning and implementation.

A second, popular intervention strategy in the school setting that does increase parental involvement is the use of school-home notes (Gimpel \& Holland, 2003). School-home notes involve the teacher recording the student's daily progress toward predetermined goals and sending the progress sheet home with the student. Typically the student earns a reward at home contingent on the performance of the school based goals. This intervention strategy is seen as efficient, feasible and effective in increasing parental involvement; however, Chafouleas, Riley-Tillman, and McDougal (2002) reviewed published investigations of school-home notes or daily behavior report cards (DBRCs) and concluded that an extensive evidence base for the effectiveness of DBRSs does not exist. The authors suggest further research of DBRCs in applied settings and investigation of the acceptability of DBRCs.

Despite the lack of strong evidence supporting school-home notes, McCain and Kelley have published a number of effective implementations of school-home note interventions (Kelley \& McCain, 1995; McCain \& Kelley, 1993, McCain \& Kelley, 1994). For example, McCain and Kelley (1993) implemented a school home note procedure with a 5-year old boy (Mitch) with ADHD to improve his noncompliant, hyperactive, oppositional behavior in the classroom. The teacher and parents developed goals for Mitch's behavior that the teacher evaluated throughout the day. The teacher provided Mitch with feedback after each rating occurred. Mitch's parents provided reinforcers contingent on his performance on the school-home note. Results indicated a significant decrease in Mitch's disruptive behavior and a significant increase in on-task behavior during intervention. The authors believed that an important component of the success of the intervention was the developmentally appropriate nature of the procedures and materials. For example, the school-home note contained pictures of expected behavior and smiley faces for the ratings of behavior, and the interval between behavior checks was short. The authors stressed the importance of tailoring an intervention to the developmental level of the child (McCain \& Kelley, 1993). Despite the positive results and the strong involvement of the family, this intervention did 
not involve the child in rating his own behavioral performance. By involving the child in the planning and implementation of the intervention the child is taught potentially powerful self-management skills.

By combining self-evaluation techniques and school-home notes, an intervention can address a young child's disruptive behavior while involving the family and increasing the child's evaluation of his/her own behavior and expectations in the classroom. By closely collaborating with the teacher, the current study attempts to bridge the research to practice gap that currently exists. Thus, the purpose of this study was to investigate the effectiveness of a combination of teacher and self-evaluation strategies and school-home note procedures to manage the disruptive behavior of young children. Another goal of the intervention was to successfully collaborate with the classroom teacher and parents to create an acceptable, teacher, child, and parent friendly intervention that blended with the teaching philosophy of the teacher and school.

\section{Method}

\section{Participants}

Participants were two Caucasian, male, kindergarten students, Craig and Nathan (ages 5 and 6 respectively), referred for disruptive classroom behavior including hyperactive, impulsive, inattentive and aggressive behavior. One kindergarten teacher with over 15 years experience as an early childhood educator, and one assistant participated in the study. The kindergarten classroom consisted of 18 children. Administrators and teachers at a university affiliated child development center referred participants to the Family Services Coordinator. Craig and Nathan were referred by their kindergarten teacher due to significant behavior problems in school and at home. According to the kindergarten teacher, they were inattentive, hyperactive, disruptive, and impulsive during school hours. According to the parents, the children were exhibiting these same behaviors at home. After the referral, the teacher and principal investigator met with the family to explain the purpose, procedures, and risks/benefits of the study, and obtain permission for participation from the parents.

Inclusion criteria were based on parent and teacher interview, and comparison with normative data using parent and teacher rating scales. Children were not selected on the basis of special class, gender, or ethnic group. Through interviews with the parents and teachers, the severity, intensity, and duration of target behaviors were operationally defined and goals for improvement were identified. Parent and/or teacher ratings on the Hyperactivity, Attention Problems or Aggression subscales of the Behavior Assessment System for Children (BASC; Reynolds \& Kamphaus, 1992) indicated that the participants' behavior was viewed as being at the clinically at-risk range (i.e., T-score greater than or equal to 60). In order to screen for possible developmental delays, the Battelle Developmental Inventory: Screening Test (BDI; Newborg, Stock, \& Wnek, 1988) was administered. The BDI measures the child's skills in the areas of Personal-Social, Adaptive, Motor, Communication, and Cognitive domains. This highly reliable and valid test yields standard scores that are rated as either consistent or inconsistent with the child's expected functioning when compared to same age peers (Newborg, et al.). Craig and Nathan were found to have age appropriate developmental skills using the BDI screener and therefore requiring no further developmental assessment. Participants were excluded from participation if they displayed the following characteristics: physical disabilities, developmental delays, gross neurological disorders, psychosis, or pervasive developmental disorder.

\section{Design}

This study used a single subject reversal design to test the effects of the interventions relative to baseline. The design included alternating baseline and intervention phases over time. By returning to baseline, the design allowed the experimental control of the intervention to be tested. The effectiveness of 
the intervention is clear if the performance changes in the desired direction when the intervention is introduced and returns to previous levels when treatment is withdrawn (Kazdin, 1982). Data were analyzed through visual inspection of the graphed data displaying changes in mean levels of performance across phases, changes in level of performance from the end of one phase to the beginning of the next phase, changes in trend or slope from one phase to the next, stability of behavior change within phases, and the latency of behavior change across phases.

The phases were as follows: Baseline (A), Intervention (B), Baseline (A), and Intervention (B). Each phase lasted approximately 1 to 3 weeks depending upon the school schedule, stability, and trend of the data. Parental permission was granted to withdraw the intervention for no more than one week to gather the second baseline data points.

\section{Dependent Measures}

Dependent measures included behavior observations of the children in the classroom that were conducted by trained graduate and undergraduate observers. The observers were not blind to the purpose of the study. Observers attempted to observe at different times during the kindergarten day. This allowed for representative observations since the intervention was implemented throughout the school day. The social behavior observation system of the Early Screening Procedure (ESP; Walker, Severson, \& Feil, 1995) was adapted to assess each child's social behavior in the classroom setting. The observational system used a partial interval system for disruptive behaviors. Disruptive behavior categories included negative social engagement, disobeying established rules, and tantrumming. (See Table 1). A behavior was coded if it occurred at any point in the 15 second interval. The observer recorded the percentage of 15-second intervals during the 15-20 minute observation that the child engaged in negative social behavior. The occurrence of each category of negative social behavior was low; therefore results were graphed using a combined percentage of disruptive behavior.

Table 1. Operational Definitions for the Direct Observations of Behavior

\begin{tabular}{ll}
\hline CATEGORY & DEFINITION \\
\hline Negative social interaction & A negative reciprocal social exchange, either verbal or \\
physical. A reciprocal exchange of social signals (e.g., \\
voice, gaze, touch) between two or more people is \\
considered negative if both parties engage in negative \\
behavior or react in a negative manner (i.e., by definition, \\
one child's "negative" behavior is only considered negative \\
when it is reciprocated by another child's "negative" \\
behavior). Negative social interaction is characterized by the \\
child saying something to another person in a derogatory \\
(e.g., "you're stupid"), uncomplimentary (e.g., "your \\
painting is yucky"), or disruptive manner (e.g., yelling \\
"Stop it" or sticking out tongue). \\
Physical aggression (e.g., hitting, kicking, or pushing). \\
The child looks away from the activity or teacher for at least \\
3 seconds. The child looks away from the activity and does \\
not follow the teachers' direction or command within 3 \\
seconds. \\
Any deviation from the rules established by the classroom \\
teacher prior to the observation. \\
The child is yelling (i.e., vocalizations above normal \\
conversational volume), kicking the floor or other inanimate \\
items, pouting and/or sulking (e.g., placing their head down
\end{tabular}


on the desk) following a negative social interaction.

Training and Interobserver Agreement. Interobserver agreement reached 95\% before initiating baseline observations. Two undergraduate students were trained in the method of data collection. Observers familiarized themselves with the operational definitions of behaviors being targeted. Interobserver agreement was based on the percentage of agreement (i.e. the number of agreements divided by the number of agreements plus the number of disagreements multiplied by 100). Two independent observers recorded child behavior during $25 \%$ of sessions throughout all phases of the study. Exact interobserver agreement ranged from $80 \%$ to $100 \%$ with an average of $89 \%$ across all categories, observers, and phases of the study. All interobserver agreement sessions were discussed with the research team to maintain high levels of agreement.

Rating Scales of Behavior. The teacher completed rating scales related to participant behavior and social skills as well as measures of intervention acceptability. The Preschool and Kindergarten Behavior Scales (PKBS; Merrell, 1994) is a 42-item questionnaire that is comprised of three social skills subscales and five-problem behavior subscales. Examples of subscales include attention problems/overactive, antisocial/aggressive and social interaction scales. Any adult that is familiar with the child is capable of completing the questionnaire (e.g., parent, caregiver, teacher). Normative data for the scale was collected from 2,855 preschool and kindergarten aged children during the years of 1992 and 1993 . The children were from 24 different U.S. communities representing the four geographical regions of the U.S. (Merrell, 1994). The $P K B S$ was collected from teachers at the end of baseline and the second intervention phase. All scales of the $P K B S$ were used for data analysis.

ADHD Rating Scale-IV (DuPaul, Power, Anastopoulos, \& Reid, 1998) is an 18-item questionnaire that requires the respondent to rate the frequency of occurrence of ADHD symptoms according to the DSM-IV-TR (APA, 2000). The respondent rates each item on a Likert scale of 0 (not at all) to 3 (very often). The teacher completed the questionnaire at the end of each baseline and the second intervention phase. The measure yields a total score and two subscale scores for dependent measures (Inattention and Hyperactivity/Impulsivity).

The teacher was provided with a checklist to remind her of the intervention procedures. The teacher completed the checklist on $20 \%$ of the classroom sessions to determine that all procedures were being followed. Teacher integrity was $100 \%$ throughout the study. The teacher would have received training and feedback if the procedural integrity measure fell below $90 \%$.

\section{Intervention Procedures}

This study involved four phases: baseline, intervention, return to baseline and intervention.

Baseline (A). Observational data were collected during the regular routine in the classroom. Eight and nine data points respectively, for Craig and Nathan, were collected across 15 school days. The teacher completed rating scales $(P K B S, A D H D-I V)$. Teachers were instructed to use all behavior management strategies typically employed in the classroom. Typically management procedures included removal from the group, discussion of behavior with a teacher and removal to the main office.

Teachers, parents, and investigators met to collaboratively design the school-home note and determine goals for the child. The team identified three goals for each child. The child then revised the goals using his or her own words to increase personalization and understanding of goals. Participants also were allowed to decorate the school-home note to further develop student interest in the intervention. Each parent's approach to reinforcement was discussed and developed during the consultation session with the primary researcher and teacher. Each parent had already established a routine of reinforcement or 
response cost in the home setting that was now linked to the school intervention. Craig's parents implemented a reward system at home that enabled Craig to earn points toward a large prize. Nathan's parents implemented a response cost procedure that involved losing a privilege if Nathan's behavior did not meet his daily goal.

Intervention (B). The intervention package consisted of 4 components. First, teachers and parents reminded participants of target behaviors and goals for improvement each morning during intervention. The teacher and student met three times a day to discuss the child's behavior. During the meetings, the teacher first encouraged each child to self-evaluate their behavior and make a judgment about their performance by assigning a frown, neutral or smiley face to each behavior goal. The child and teacher then discussed the child's evaluation of his behavior. The teacher then provided the child with her evaluation of the behavior and guided the child to understand the teacher's view of the child's behavior. The teacher's judgment of behavior was charted on the child's daily note. At the end of the day, teachers and students added the number of smiley faces and determined if the child had met his goal. The note was then sent home to the child's parents. At home, parents applied consequences based on their child's performance during school. Throughout the intervention, the parents were kept informed of their child's progress through the daily school home notes, as well as communication with the teacher and primary investigator. Teachers continued to use typical classroom discipline procedures for disruptive, noncompliant, or aggressive behavior such as removal from the activity or individual discussion with the teacher.

Return to baseline (A). The intervention was withdrawn from the classroom. Participants were informed that the school-home note would not be used. Teachers were instructed to continue to use all behavior management strategies that were typically used in the classroom.

Intervention $(B)$. The teacher and student self-evaluation and school home note were once again implemented in the classroom. Parents and teachers followed the same procedures in the first phase of intervention.

\section{Results}

\section{Craig}

As indicated by pretreatment parent interview, teacher interview and ratings, direct observation, and past school records, Craig's disruptive behavior had been a significant problem in the classroom since preschool. Given the stability of problem behavior, the school-home note was implemented with the goal of behavior management in the classroom, communication between parent and teacher and beginning self-management of Craig's behavior. Craig's baseline data indicated an increasing trend in disruptive behaviors with a mean of 32.63 intervals (see Figure 1). After implementation of the intervention, Craig's disruptive behaviors immediately decreased to a mean of 6.57 intervals. After the intervention reduced disruptive behavior, it was withdrawn to experimentally test the effects of the intervention. Craig's disruptive behavior returned with an increasing trend and a mean of 25.33. When the intervention was reinstated, Craig's behavior immediately decreased to a mean of 2.5. Due to illness and the end of the school year, only two data points could be collected for this intervention phase. Teacher ratings on the $P K B S$ of Craig's behavior in the classroom indicate baseline levels of Problem Behaviors and Social Skills at a standard score of 117 and 73, respectively (see Table 2). After intervention, teacher ratings of Problem Behaviors decreased to a standard score of 107 and ratings of Social Skills increased to a standard score of 90 . On the $A D H D-I V$, teacher ratings at baseline indicated a percentile score of 98 for Inattention, 87 for Hyperactivity/Impulsivity and 94 for the Total Score. After the final intervention, his ratings decreased slightly to a percentile score of 86 for Inattention, 75 for Hyperactivity/Impulsivity and $<84$ but $>80$ for the Total Score. 


\section{Craig}

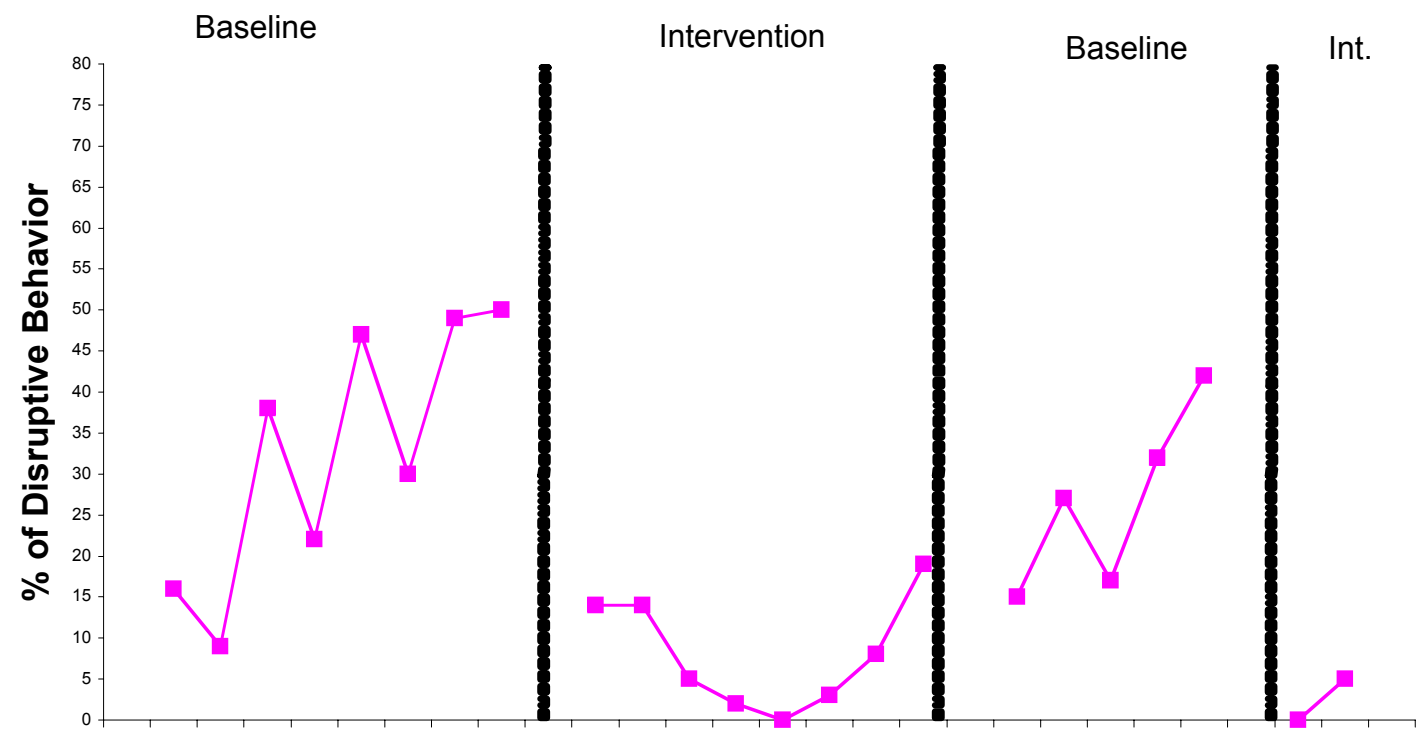

Observation Sessions

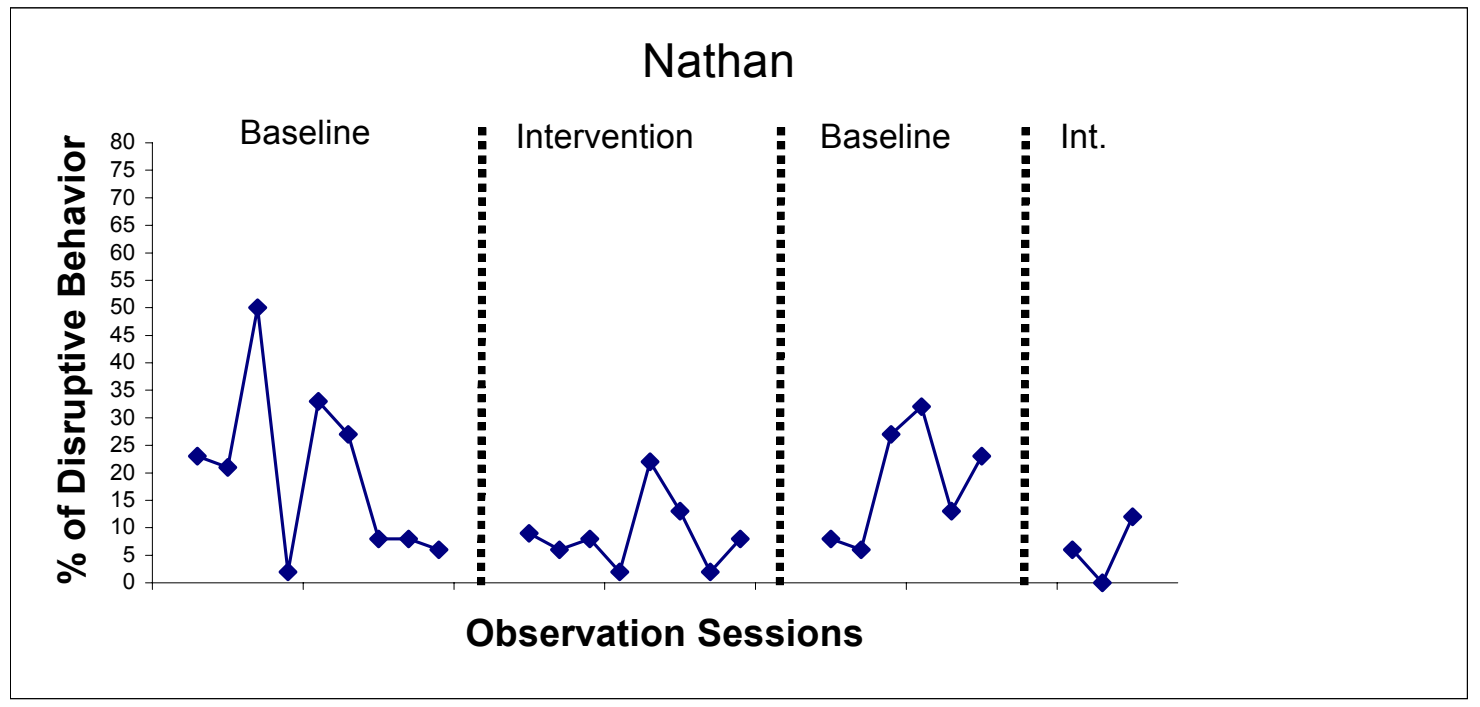

Figure 1. Craig's and Nathan's Percentage of negative social behavior as a function of observations sessions.

Table 2. Teacher Ratings on the Preschool and Kindergarten Behavior Scales and ADHD Rating Scale-IV

\begin{tabular}{lcccc} 
& & Nathan & Craig \\
& Baseline & Intervention & Baseline & Intervention \\
\hline PKBS $^{*}$ & & & & 90 \\
Social Skills & 85 & 102 & 73 & 107 \\
Problem & 132 & 102 & 117 & \\
Behavior & & & &
\end{tabular}




\begin{tabular}{|c|c|c|c|c|}
\hline \multicolumn{5}{|l|}{ ADHD IV** } \\
\hline Inattention & $<75 \%$ & $<75 \%$ & $98 \%$ & $86 \%$ \\
\hline Hyp/Impuls & $<75 \%$ & $<75 \%$ & $87 \%$ & $75 \%$ \\
\hline Total Score & $<75 \%$ & $<75 \%$ & $94 \%$ & $<84 \%$ \\
\hline
\end{tabular}

\section{Nathan}

After analyzing screening measures, discussing Nathan's behavior with his parents and teachers, and observing in the classroom, it was apparent that Nathan's disruptive behavior was influencing his performance in the classroom and home setting. His disruptive behavior was reported by both parent and teacher to be chronic, yet inconsistent. During baseline, Nathan's mean percentage of disruptive behaviors was 19.78. Nathan's percentage of disruptive behaviors was inconsistent throughout the baseline phase and produced a downward trend (see Figure 1). This variability of behavior was consistent with parent and teacher reports. Given his past history of unpredictable behavior in the classroom, with no stable improvement, the intervention was implemented despite the downward trend in data. After implementation of the intervention Nathan's negative behaviors in the classroom became more stable, reduced to a mean of 8.75 and produced a fairly low and flat trend line.

To test the effects of the intervention, the school-home note was briefly withdrawn from use in the classroom. Nathan's disruptive behavior gradually increased to a mean of 18.17. Once reinstated, Nathan's behavior decreased once again to a mean of 6 .

Nathan's teacher completed the $P K B S$ and $A D H D-I V$ during the first baseline and second intervention phase (see Table 2). During baseline ratings on the PKBS of Nathan's Social Skills and Problem Behaviors received a standard score of 85 and 132, respectively, indicating a significant deficit in social skills and significantly elevated levels of disruptive behaviors in the classroom. After the school-home note intervention, Nathan's teacher rated his Social Skills and Problem Behaviors as improved with both scales receiving standard scores of 102. There was no difference in teacher report of $A D H D$ symptoms based on the $A D H D-I V$ between baseline and intervention.

Treatment Acceptability. At the conclusion of the study the teacher was asked to complete a teacher satisfaction rating, the Intervention Rating Profile - 15 (IRP-15; Martens, Witt, Elliott, \& Darveaux, 1985). The $I R P-15$ is a 15 -item rating scale designed to measure the acceptability of classroom interventions using a 5-point Likert scale ranging from 1 (not acceptable) to 5 (very acceptable). It has adequate psychometric properties (Martens, et al.). Teacher ratings on the IRP-15 ranged from 4 to 6 with a mean rating of 5.6, indicating high acceptance of the intervention procedures.

\section{Discussion}

The results of this study indicate that self-evaluation and school home notes were an effective intervention package for managing the disruptive behavior of two kindergarten boys. Both participants displayed decreased levels of disruptive behavior in the classroom. Behavioral observations showed a decrease in disruptive behavior during intervention when compared to baseline. Craig's response to intervention was more stable and durable, whereas, Nathan exhibited an overall decrease in disruptive behavior but his behavior remained variable throughout the study. In addition, the procedures attempted to incorporate the beginning steps to self-management of behavior. For example, each child became an active participant in the intervention by changing the goal statements to his own words and decorating the school-home note, and the procedure encouraged the children to evaluate their behavior. The kindergarten teacher involved in the study found the intervention to be effective and acceptable through acceptability ratings. 
Furthermore, this study highlights the importance of collaboration between investigators, teachers, students, and parents. As a team, the investigators, teacher, and parents designed the schoolhome note and child's goals based on the classroom environment, teaching philosophy, specific child behaviors and family needs. The child participated by rewording the goals into developmentally appropriate and familiar language and by personalizing the school-home notes by decorating them. This appeared to create a sense of ownership and investment in the intervention. During the intervention, the teacher frequently contacted the investigators and parents to facilitate communication of intervention progress and procedures. Collaboration appeared to contribute to the success of the intervention.

Implications for Practice

As evidenced by this study, self-evaluation and school-home notes may be an effective, developmentally appropriate intervention package to manage disruptive behavior of young children. Given the collaborative nature of the design, the high acceptability ratings by the teacher, and the willingness of the parents to participate in the intervention planning process, this intervention could be implemented by practitioners in other school settings. The following components of the intervention lend to the practical nature of the intervention. First, teachers rated the intervention as non-intrusive on class time or resources. The behavior checks throughout the day require only a few minutes to accomplish and can be timed during natural transition periods. In addition, the only materials needed for implementation are the copies of the home note. Second, by parents providing consequences in the home setting, the chance that other students will be influenced by a child receiving differential consequences for behavior is decreased. Third, the school home note can serve as a progress monitoring method. For example, the teacher may keep track of the number of goals the child has met each day. That number is then analyzed for improvement. This also allows for evaluation of progress if modifications are warranted.

Based on anecdotal notes and clinical impressions, practitioners should pay particular attention to the following points when implementing this intervention package. First, teachers and parents should be willing to collaborate and follow through with the intervention. Second, the length of time between behavior checks needs to be tailored to each participant and may fluctuate depending on the activities of the day and the child's goals. Third, teachers and parents must be vigilant about reminding the students of their goals. Finally, parents must implement the child's consequences with immediacy and consistency. The above points were noted by the investigators and teacher as integral but unfortunately no data are available to support these claims. Future research may want to investigate these particular components of the intervention through component analyses. In addition, some children may need more intensive, comprehensive, immediate consequences for their disruptive behavior. Practitioners may want to consider school-home notes as a first line of treatment and if unsuccessful conduct a functional behavioral assessment of the disruptive behavior and implement a more comprehensive intervention package based on the function of the behavior.

As the intervention progressed, the teacher noted that there appeared to be an awareness developing in the participants in support of the self-management nature of the intervention. For example, near the end of intervention, the teacher reported that she could simply look at the child with a contemplative look and the child would stop the behavior. Previous to intervention, the participants would not have understood this nonverbal redirection. It appeared that the child had become more aware of his behavior, and needed fewer teacher cues to stay on track. Therefore, this intervention provided teachers with a structure to communicate with the children about their disruptive behavior, possibly decreasing negative interactions between the child and teacher. In addition, the teachers reported feeling an increased positive relationship with each child by the consistent praise and reminders of goals. It was reported by the teacher that a sense of community, caring and collaboration was fostered between the child and teacher. 


\section{Implications for Research}

Previous research found self-management and school-home notes to be successful with students with academic and behavior problems (McCain \& Kelley, 1993; Workman, et al, 1982); however, the case study nature limited generalizability of the results (McGoey, Eckert, \& DuPaul, 2002). This study replicated the findings of this previous work by successfully decreasing the disruptive behavior of two kindergarten students. In addition, the study extended the previous literature by implementing the intervention in a kindergarten classroom, creating developmentally appropriate procedures that matched the teaching philosophy of the kindergarten classroom and teacher, and increasing the collaboration between teacher and parent. As McCain and Kelley state, designing a developmentally appropriate intervention appears to be an important variable in intervention effectiveness.

Given the small sample size of this study, future research should focus on replicating this work with more children, younger children, children in diverse settings and with diverse backgrounds. Investigations should include follow-up data, procedural integrity checks at home, and functional assessment of the child's behaviors to aid in goal development.

\section{Limitations}

Given the applied nature of this work, the intervention is not without limitations. Specifically, the observational data is highly variable. However, one of the goals of this project was to implement research in the classroom. Therefore, scientific decisions were often overridden by clinical decisions. For example, an intervention was implemented when the teachers requested even though the baseline data was trending toward improvement of the behavior for Nate. In addition, the intervention included many components and could not control for basic classroom variables such as increased teacher praise. Therefore, future research should attempt to better isolate these variables through component analysis.

The results of this intervention should be interpreted with caution until further replication of findings. The study involved only two participants with one teacher at a private, university based school. The investigation did not include a compliance check on the parents' implementation of the consequences. Both sets of parents indicated reluctance to this data collection. The teacher integrity to the implementation of the intervention was rated as $100 \%$, however, this may be an overestimate because it was a self-report of compliance. Also, the teacher ratings of behavior change could not be analyzed statistically, instead relying on visual description of change that may not account for regression to the mean, practice effects or teacher bias created by investment in the intervention. Teacher ratings of Nathan's behavior on the ADHD-IV remained relatively stable after intervention. This was not surprising given the daily and weekly variability found in Nathan's behavior.

In addition, the second phase of intervention was extremely short due to the end of the school year limiting the validity of the results. Furthermore, no follow-up was possible because both children did not attend the facility in the summer and changed schools for the following academic year, thus limiting the investigators knowledge of the long-term impact of the intervention.

\section{Summary}

Overall, school home notes appear to be a promising intervention to manage the disruptive behavior of children in early childhood. The procedures are non-intrusive, require limited amounts of teacher time and resources, and are acceptable to both teachers and students. In addition, the intervention may promote collaboration between the teachers, parents, children, and school psychologist. This 
collaboration fosters communication, a commitment to the behavior change and investment in the intervention that adds to the success of the intervention.

\section{References}

American Psychiatric Association. (2000). Diagnostic and statistical manual of mental disorders (4th ed, Text Revision.). Washington, D.C.: Author.

Barkley, R.A. (1998). Attention Deficit Hyperactivity Disorder: Handbook of diagnosis and treatment, (2nd Ed). New York: Guilford Press.

Chafouleas, S.M., Riley-Tillman, T.C., \& McDougal, J.L. (2002). Good, bad, or in-between: How does the daily behavior report card rate? Psychology in the Schools, 39, 157-169.

DuPaul, G. J. \& Eckert, T. L. (1997). The effects of school-based interventions for attention deficit hyperactivity disorder: A meta-analysis. School Psychology Review, 26, 5-27.

DuPaul, G. J., McGoey, K. E., Eckert, T. L., \& Van Brakle, J. (2001). Preschool children with Attention-Deficit/Hyperactivity Disorder: Impairments in behavioral, social, and school functioning. Journal of the American Academy of Child and Adolescent Psychiatry, 40, 508 515.

DuPaul, G. J., Power, T. J., Anastopoulos, A. D. \& Reid, R. (1998). AD/HD Rating Scale IV: Checklists, Norms, and Clinical Interpretation. New York: Guilford Press.

DuPaul, G.J., \& Stoner, G. (2003) ADHD in the Schools: Assessment and Intervention Strategies. New York: Guilford Press.

Gimpel, G.A., \& Holland, M.L., (2003). Emotional and Behavioral Problems of Young Children: Effective Interventions in the Preschool and Kindergarten Years. New York: Guilford Press.

Kazdin, A. E. (1982). Single-Case Research Designs: Methods for Clinical and Applied Settings. New York: Oxford University Press.

Kelley, M.L. \& McCain, A.P. (1995). Promoting academic performance in inattentive children: The relative efficacy of school-home notes with and without response cost. Behavior Modification, $19,357-375$.

Kratochwill, T. R., \& Stoiber, K. C. (2000). Empirically supported interventions and school psychology: Conceptual and practices issues-Part II. School Psychology Quarterly, 15, 233-253.

Martens, B. K., Witt, J. C., Elliott, S. N., \& Darveaux, D. X. (1985). Teacher judgements concerning the acceptability of school-based interventions. Professional Psychology: Research and Practice, 16, 78-88.

McCain, A. P. \& Kelley, M. L. (1993). Managing the classroom behavior of an ADHD preschooler: The efficacy of a school home note intervention. Child and Family Behavior Therapy, 15, 33-44.

McCain, A.P. \& Kelley, M.L. (1994). Improving classroom performance in underachieving preadolescents: The additive effects of response cost to a school-home note system. Child and Family Behavior Therapy, 16, 27-40. 
McGoey, K. E., Eckert, T. L., \& DuPaul, G. J. (2002). Interventions for preschoolers with ADHD: A literature review. Journal of Emotional and Behavioral Disorders, 10, 14-28.

Merrell, K. W. (1994). Preschool and Kindergarten Behavior Scales. Brandon, VT: Clinical Psychology Publishing Company.

Mooney, P. Ryan, J.B., Uhing, B.M., Reid, R, \& Epstein, M.H. (2005). A review of self-management interventions targeting academic outcomes for students with emotional and behavioral disorders. Journal of Behavioral Education, 14, 203-221.

Newborg, J., Stock, J. R., \& Wnek, L. (1988). Manual for the Battelle Developmental Inventory. Boston, MA: Houghton Mifflin.

Pelham, W. E., Wheeler, T., \& Chronis, A. (1998). Empirically supported psychosocial treatments for Attention Deficit Hyperactivity Disorder. Journal of Clinical Child Psychology, $27,156-167$.

Reid, R. Trout, A.L., \& Schartz, M. (2005). Self-regulation interventions for children with Attention Deficit/Hyperactivity Disorder. Exceptional Children, 71, 361-377.

Reynolds, C. R., \& Kamphaus, R. W. (1992). Behavioral Assessment System for Children. Circle Pines: $\mathrm{MN}$ : American Guidance Service.

Shapiro, E. S. \& Cole, C. L. (1994). Behavior change in the classroom: Self-management intervention strategies. New York: Guilford.

Stoiber, K. C., \& Kratochwill, T. R. (1999). Empirically supported interventions and school psychology: Rationale and methodological issues-Part I. School Psychology Quarterly, 15, 75-105.

Walker, H. M., Severson, \& Feil, E. G. (1995). Early screening project: A proven child find process. Longmont, $\mathrm{CO}$ : Sopris West.

Workman, E. A., Helton, G. B., \& Watson, P. J. (1982). Self-monitoring effects in a four-year-old child: An ecological behavior analysis. Journal of School Psychology, 20, 57-64.

\section{Author Contact Information:}

Kara E. McGoey, Ph.D., Duquesne University, School Psychology Program, 209A Canevin Hall, Pittsburgh, PA 15282. Email: $\underline{\text { mcgoeyk@duq.edu }}$

Authors note: The authors would like to thank the teachers and children at the Child Development Center, Kent, Ohio for their participation in this project. 